\title{
A Low Complexity Transmitter Structure for OFDM-FDMA Uplink Systems
}

\author{
Dirk Galda and Hermann Rohling \\ Technical University Hamburg-Harburg, Department of Telecommunications \\ Eißendorfer Straße 40, 21073 Hamburg, Germany \\ Elena Costa, Harald Haas and Egon Schulz \\ SIEMENS AG, ICM N MR ST 8 \\ Werner-von-Siemens-Ring 20, 85630 Grasbrunn, Germany
}

\begin{abstract}
The orthogonal frequency division multiplex (OFDM) transmission technique can efficiently deal with the effects of multi-path propagation in the broadband radio channel. It also has a high system inherent flexibility for designing a multiple access scheme by combining the conventional TDMA, FDMA and CDMA approaches with the OFDM modulation scheme. The FDMA multiple access scheme is especially interesting for an uplink of a communication system since it can completely avoid any multiple access interferences (MAI). Moreover, the peak-to-average ratio (PAR) of the uplink OFDM transmit signal can be greatly reduced if this OFDM-FDMA multiple access scheme is additionally combined with a data spreading technique based on a Discrete Fourier Transform (DFT) spreading matrix using only the user specific subcarriers. Since the DFT spreading operation and the IDFT operation used as a part of the OFDM modulation scheme cancel out each other the complexity of the transmitter structure for an OFDM-FDMA uplink can be greatly reduced.
\end{abstract}

Keywords-OFDM, FDMA, CDMA, Uplink, PAR, DFT Spreading

\section{INTRODUCTION}

The broadband radio channel is characterized by its frequency selective fading due to multi-path propagation. In mobile communications applications the radio channel is additionally time-variant due to the movement of the mobile terminal. The orthogonal frequency division multiplexing (OFDM) transmission technique can cope with the effects of frequency selectivity and time variance with a low implementation and computation complexity. Inter symbol interferences (ISI) as well as inter carrier interferences (ICI) can be completely avoided by dividing the total system bandwidth into a large number of spectrally overlapping but mutually orthogonal non-frequency selective narrow-band subchannels and by introducing an additional guard interval into the OFDM symbol. Even at the output of a frequencyselective channel this orthogonality of the subcarriers can be maintained. The equalization of each subchannel is then reduced to a single complex multiplication per subchannel [1].

Recently, the combination between the classical multiple access schemes "TDMA", "FDMA" and "CDMA" and the OFDM transmission technique have been intensively discussed [2]. For the synchronous downlink MC-CDMA or OFDM-CDMA can offer a good performance but due to the loss of orthogonality of the used codes in a frequency selective radio channel the receiver of a mobile terminal has to cope with MAI by single or multi user detection (SUD, MUD) scheme. The effect of MAI is even increased if OFDM-CDMA is used in the uplink due to the independent channels which affect the transmission of different mobile terminals.

The subdivision of the transmission bandwidth into a set of orthogonal subcarriers can additionally be exploited by an OFDM-FDMA multiple access scheme. By allocating distinct sets of subcarriers to different users the available bandwidth can be flexibly shared between different mobile terminals while avoiding any multiple access interferences (MAI) between different users. The FDMA multiple access scheme offers not only a high flexibility for the radio resource management (RRM) but can also increase the bandwidth efficiency of the complete system by avoiding the use of highly attenuated subcarriers for specific users based on the knowledge of the channel transfer function [2]. Moreover, the OFDM-FDMA multiple access scheme can be adapted to the measured radio channel knowledge at the transmitter site using bit-loading techniques. If channel state information is not available the performance can be increased using an additional spreading over the subcarriers assigned to one user. The resulting computation complexity of the total system can by this means be adopted to the given system requirements which is especially of importance for the mobile terminal and in the uplink case. The alternative OFDM-FDMA multiple access scheme is therefore of importance for the uplink case and can be advantageous over OFDM-CDMA schemes because of its ability to avoid MAI if ideal carrier synchronization is assumed for all mobile terminals and the base station.

Since the OFDM transmit signal results from the superposition of a large number of independent data symbols the envelope of the complex baseband time signal has in general a large peak-to-average ratio (PAR). The largest output power value of the amplifier will limit the maximum amplitude of the signal in the transmitter. Therefore, non-linear distortions due to clipping and amplification effects in the transmit signal will lead to both in-band and out-of-band emissions. In the past different techniques for reducing the PAR by changing the transmit signal independently from knowledge of other parts of the OFDM transmitter have been developed. But the PAR of the transmit signal envelope which employs an OFDM-FDMA multiple access scheme can significantly be reduced if additional spreading techniques are applied which spread the user data over the allocated subcarrier only. In this case an appropriate spreading technique must be designed re- 


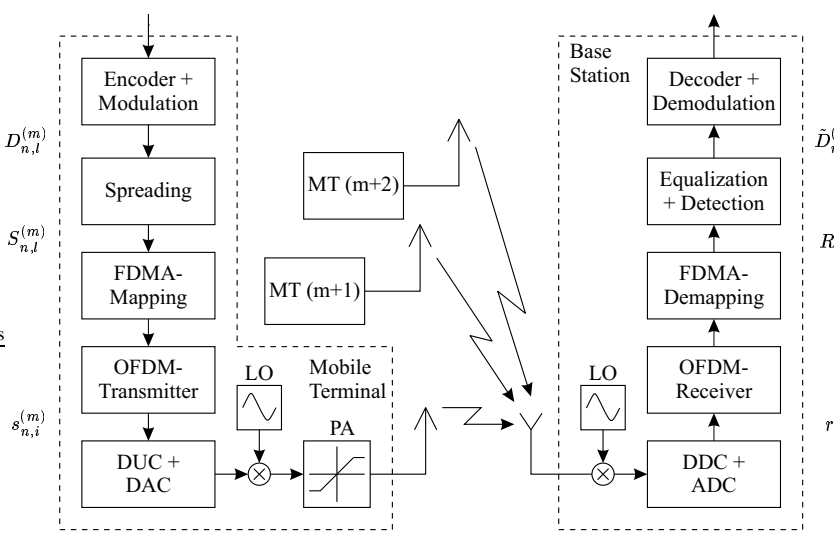

Fig. 1. Baseband system model for the OFDM-FDMA uplink with individual spreading of user data

ducing the PAR to a minimum value. It will be shown in this paper that using the Discrete Fourier Transform (DFT) matrix as an orthogonal spreading technique will reduce the PAR significantely [3]. Furthermore, the DFT based spreading operation and the IDFT based OFDM modulation technique cancel out each other which means that the transmitter structure can be simplified to a single carrier transmitter with an additional guard interval in this specific case which helps to reduce the computational complexity of the transmitter of the mobile terminal.

This paper shows how an OFDM-FDMA uplink system can be combined with a user data spreading technique based on the DFT spreading matrix to reduce the PAR of the uplink signal without increasing the computational complexity of the transmitter. In Section II the structure of the analyzed OFDM-FDMA system combined with a DFT spreading matrix is described for the uplink application. In Section III the influence of a DFT matrix applied for data spreading is analyzed for an OFDM-TDMA multiple access scheme and is extended to an OFDM-FDMA system in a separate subsection. The general topic of non-linearities in an OFDM system is reviewed in Section IV. Quantitative results are given in Section V and SectionVI summarizes the papers content.

\section{System StRucture}

The baseband system model of an OFDM-FDMA uplink with individual spreading of user data is shown in Fig. 1. In this case $M$ different users are considered and each user allocates $L$ different subcarrier exclusively. The total number of subcarrier in the considered transmission system is $N_{C}=L \cdot M$. The input data stream for each mobile user $m$, $m=0, \ldots, M-1$, is convolutionally encoded in a first step. The bit sequence is then mapped onto $L$ complex modulation symbols $D_{l}^{(m)}, l=0, \ldots, L-1$, of a coherent, higher-level modulation scheme. The $L$ modulation symbols are spread over the $L$ user specifically allocated subcarrier with an unitary spreading matrix $[\boldsymbol{C}]$ resulting in $L$ complex transmit symbols $S_{l}^{(m)}$. The spreading operation can be denoted mathematically by the following simple matrix multiplication

$$
\vec{S}^{(m)}=[\boldsymbol{C}] \vec{D}^{(m)}
$$

where each complex transmit symbol $S_{l}^{(m)}$ is calculated by the sum of $L$ user modulation symbols $D_{l}^{(m)}$ weighted by $L$ orthogonal code vectors $\vec{C}_{l}=\left(C_{l, 0}, C_{l, 1}, \ldots, C_{l, l}\right)$ with $l=0, \ldots, L-1$

$$
S_{l}^{(m)}=\sum_{\nu=0}^{L-1} C_{l, \nu} D_{\nu}^{(m)} \quad \text { for } \quad l=0, \ldots, L-1 .
$$

The transmit symbols $S_{l}(m)$ are then mapped onto $L$ of the available $N_{C}$ subcarrier which are exclusively allocated to user $m$. In principle, the set of subcarrier assigned to each user can be composed of any $L$ out of $N_{C}$ subcarrier that have not been assigned to another user.

If a spreading technique is employed including the user specific subcarriers and the spreading gain should be maximized then the selected subcarrier set should be more or less uncorrelated [4]. Therefore in this paper a subset is considered where the selected subcarrier are placed on equidistant subcarriers over the entire bandwidth, see Fig. 2. In this case
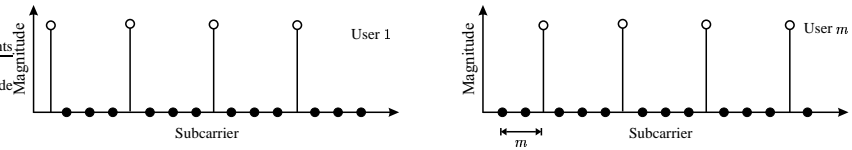

Fig. 2. User $m$ allocates the equidistant subset of subcarriers shift by $m$ subcarriers in the frequency domain

the selected subcarrier set can be assumed to be mutually independent which leads to a maximum spreading gain, the subcarrier selection is exclusive for each user, which avoids any MAI, and the signalling overhead is minimized. Furthermore, it is assumed in the sequel that the set of equidistant subcarrier are shifted by $m$ subcarrier in the frequency domain if the subset is assigned to user $m$. The proposed subcarrier selection and modulation process does not need any radio channel state information in this case.

Independent of the considered spreading matrix $C$ but assuming the equidistant allocation of subcarrier over the entire bandwidth the resulting OFDM time signal of user $m$ can analytically be described as

$$
\begin{gathered}
s_{i}^{(m)}=e^{j 2 \pi i m / N_{C}} \frac{1}{\sqrt{L}} \sum_{l=0}^{L-1} S_{l}^{(m)} e^{-j 2 \pi i l / L} \\
\text { for } i=-N_{G}, \ldots, 0, \ldots, N_{C}
\end{gathered}
$$

where $N_{G}$ denotes the length of the guard interval which is inserted into the transmit time signal.

At the base station the received time signal in the base band is processed by removing the guard interval, calculating the Fourier transformation (FFT) which splits the time signal into the orthogonal subcarrier channels and finally selecting the user specific complex valued symbols $R_{l}^{(m)}$ at the FFT output. The user specific subcarrier set is located at subcarrier $k=m, m+M, \ldots, m+(L-1) M$ for user $m$. The complex valued symbols $R_{l}^{(m)}$ can be described as

$$
R_{l}^{(m)}=H_{m+l M}^{(m)} S_{l}^{(m)}+N_{m+l M}^{(m)} \cdot
$$


where $H_{k}^{(m)}$ denotes the complex channel transfer factor on subcarrier $k$ of user $m$ and $N_{k}^{(m)}$ the complex Gaussian noise with variance $\sigma_{N}^{2}$. For a perfectly time and carrier synchronized system the received symbols are not influenced by ISI and ICI at all.

An estimate of data symbols $\tilde{D}_{l}^{(m)}$ can then be calculated independently for each user by applying any single- or multicode detection techniques.

\section{DFT SPREADING}

The spreading matrix $[\boldsymbol{C}]$ of an OFDM-FDMA system is often only characterized by its frequency diversity properties. It therefore is chosen to be unitary in order to make the detection process easier, to distribute the signal energy of the superimposed code symbols uniformly over all subcarrier and not to change the distance of the code vectors. Thus, in many OFDM systems with additional spreading the Walsh-Hadamard (WH) matrix is employed since it has the additional advantage that it only consists of only " +1 " and "1 " elements which can reduce the computational complexity. The discrete Fourier matrix is also unitary but additionally has an influence on the PAR of an OFDM transmit signal. In this Section the influence of such an Fourier speading matrix on the resulting PAR will be discussed.

\section{A. OFDM-TDMA with DFT Spreading}

In [3] the Discrete Fourier Transformation (DFT) matrix has been used for spreading the vector $\vec{D}$ of length $L=N_{C}$ over all subcarriers of an OFDM system. If a single user is assumed in this OFDM system the spreading operation includes all subcarrier within the entire bandwidth. In this case the DFT spreading matrix and the IDFT operation in the OFDM modulation process cancel out each other. The OFDM transmitter structure with DFT spreading matrix is therefore technically reduced to the serial sequence of complex transmit data symbols $D_{i}$ to which a guard interval is added in the time domain as a cyclic prefix

$$
s_{i}^{(1)}=D_{i \bmod N_{C}} \text { for } i=-N_{G}, \ldots, 0, \ldots, N_{C}
$$

where mod denotes the modulo operation, as shown in Fig. 3.

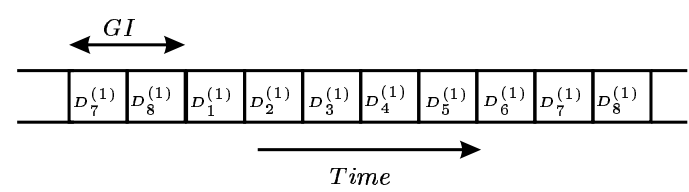

Fig. 3. Time signal of an OFDM-TDMA system with DFT spreading

Since the transmit signal of this OFDM system consist of a sequence of complex modulation symbols $D_{i}$ it has the same PAR as the modulation scheme employed for single carrier transmission techniques.

After calculating the FFT in the receiver which splits the received time signal into the orthogonal sub-channel the same single- or multi-code detection techniques can be applied which are well known from OFDM-CDMA receiver structures. The same bit error rate (BER) performance compared to a system using a Walsh-Hadamard spreading matrix can therefore be achieved if a DFT spreading matrix is considered instead. But in case of a DFT spreading matrix the resulting PAR is significantly reduced. The general structure of an OFDM transmitter including DFT spreading is shown in Fig. 4.

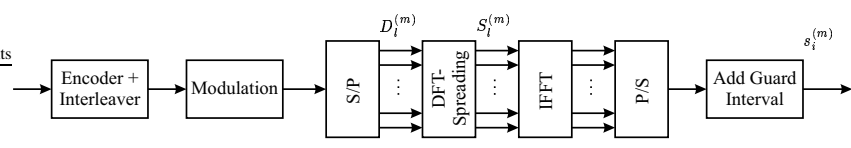

(a) OFDM transmitter including DFT spreading over all subcarriers

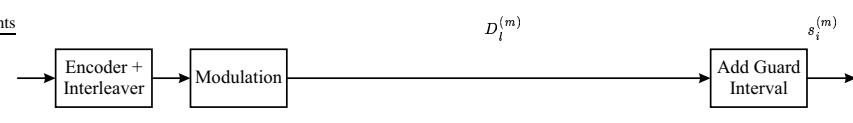

(b) Realistic but simplifi ed OFDM transmitter structure

Fig. 4. In an OFDM system including a DFT spreading matrix and an IFFT for the OFDM modulation process both matrices cancel out each other.

\section{B. OFDM-FDMA with DFT Spreading}

The advantage of a DFT spreading matrix can also be exploited in the uplink of an OFDM-FDMA system. If the user data is spread only over $L<N_{C}$ subcarriers then the DFT of length $L$ used for spreading does not directly cancel out with the length $N_{C}$ IDFT of the OFDM modulator in general. Only when the spreaded symbols $S_{l}^{(m)}$ are mapped onto equidistant located subcarriers with a spacing of $N_{C} / L=M$ the DFT spreading and the OFDM modulation can be removed in the transmitter structure. This can be seen by inserting Equation (2) into Equation (3) using the elements of the discrete Fourier matrix

$$
C_{i, j}=\frac{1}{\sqrt{L}} \cdot e^{j 2 \pi i j / L}
$$

the transmit signal of user $m$ can be written as

$$
\begin{aligned}
s_{i}^{(m)} & =e^{j 2 \pi i m / N_{C}} \frac{1}{\sqrt{L}} \sum_{l=0}^{L-1} \sum_{\nu=0}^{L-1} C_{l, \nu} D_{\nu}^{(m)} e^{-j 2 \pi i l / L} \\
& =e^{j 2 \pi i m / N_{C}} \frac{1}{L} \sum_{\nu=0}^{L-1} D_{\nu}^{(m)} \underbrace{\sum_{l=0}^{L-1} e^{j 2 \pi(\nu-i) l / L}}_{=L \delta_{L}(\nu-i)} \\
& =e^{j 2 \pi i m / N_{C}} \sum_{\nu=0}^{L-1} D_{\nu}^{(m)} \delta_{L}(\nu-i) \\
s_{i}^{(m)} & =e^{j 2 \pi i m / N_{C}} D_{i \bmod L}^{(m)}
\end{aligned}
$$

for $i=-N_{G}, \ldots, 0, \ldots, N_{C}$ and the periodic dirac pulse sequence $\delta_{L}()$. The transmit time signal $s_{i}^{(m)}$ of user $m$ in an OFDM-FDMA uplink using DFT spreading matrix and an equidistant subcarrier allocation results therefore in a periodic repetition of the complex user data symbol $D_{l}^{(m)}$ sequence including an added guard interval as cyclic prefix, 


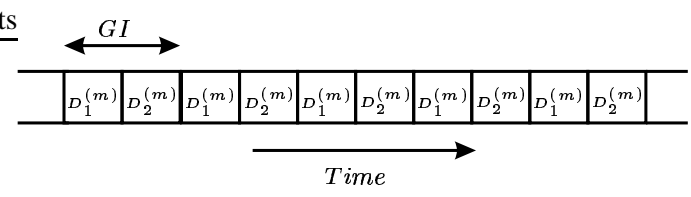

Fig. 5. Time signal of an OFDM-FDMA uplink system with DFT spreading is characterized by a periodic repetition $\left(N_{C}=8, M=4\right)$

shown in Fig. 5. This periodic data sequence is multiplied by a user specific complex signal $e^{j 2 \pi i m / N_{C}}$ due to the user individual frequency shift by $m$ subcarriers of the complete allocated subcarrier set. The number of periods inside a single OFDM symbol is equal to the spacing $M$ of the subcarriers allocated to user $m$.

The structure of the OFDM transmitter can be simplified by this approach since both the DFT spreading operation and the IFFT calculation of the conventional OFDM transmitter cancel out and can be removed in the technical realization completely and will be replaced by a simple repetition process of the considered user data $D_{l}^{(m)}$. The simplified transmitter structure is depicted in Fig. 6. Any equidistant subcar-

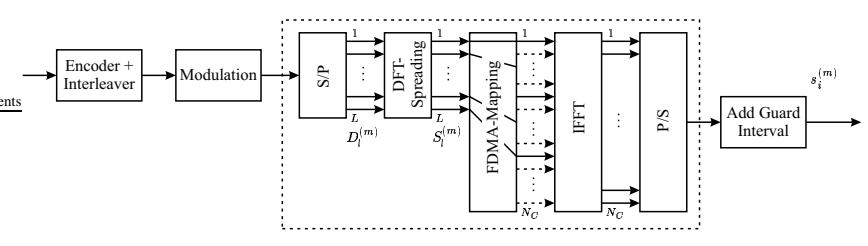

(a) OFDM-FDMA transmitter with DFT spreading over equidistant subcarriers

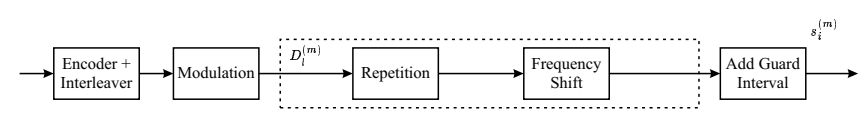

(b) Realistic but simplifi ed OFDM transmitter structure

Fig. 6. In an OFDM-FDMA uplink system including DFT spreading matrix applied to a set of equidistant subcarriers the DFT spreading matrix and the OFDM IFFT transformation cancel out each other.

rier allocation can be used with the proposed FDMA scheme since it only influences the number of periods of the transmit signal. Therefore the user data rate can be flexibly adjusted by assigning the required number of subcarriers to each user.

With the same argumentation the PAR can also be reduced for the OFDM-FDMA downlink even though not the same simple transmitter structure can be used. The PAR of the time signal is in this case determined by the number of users $M=N_{C} / L$ instead of the number of used subcarrier and will therefore in most cases be lower than for a conventional OFDM-FDMA system without additional spreading.

Since the time signal of the OFDM-FDMA uplink with DFT spreading is identical to a single carrier transmission additional techniques to reduce the PAR originally developed for single carrier systems like the $\pi / 4$-QPSK can be employed.

\section{NON-LINEARITIES}

For a sufficiently large number $L$ of used subcarrier per user the time discrete OFDM signal $s_{i}^{(m)}$ of user $m$ given in Equation (3) has an approximately Gaussian amplitude distribution due to the superposition of statistically independent modulation symbols $S_{l}^{(m)}$ on all used subcarriers. The maximum peak-to-average ratio (PAR), defined by

$$
P A R=10 \log _{10}\left(\frac{\max \left(|s(t)|^{2}\right)}{\sigma_{s}^{2}}\right)[d B]
$$

where $\sigma_{s}^{2}$ denotes the average transmit power, occurs only if the identical modulation symbol $S_{l}$ is transmitted on all used subcarriers. In this case the PAR is determined by the number of used subcarriers $L$.

A non-linear device is often modelled by a soft-limiter described by its non-linear amplitude modulation and phase modulation characteristic as

$$
g(a)= \begin{cases}a & |a| \leq A_{0} \\ A_{0} & \text { else }\end{cases}
$$

where $a$ is the magnitude of the time signal and $A_{0}$ defines the maximum output amplitude of the device.

When the OFDM time signal is passed through a nonlinear device as the high power amplifier of the transmitter large signal amplitudes will be clipped by that devices if they exceed its range of linear operation. A non-linear signal distortion will cause both in-band and out-of-band emissions of the transmit power spectrum. In-band distortions will only degrade the performance of the OFDM system whereas outof-band emissions will also disturb services in adjacent transmission bands. Especially out-of-band emissions have therefore to be reduced below a certain power level to comply with a given spectrum mask. Additionally, because of identical transmission bands of different users in an OFDM-FDMA uplink system in-band emissions will lead to multiple access interferences (MAI) between different users.

In order to avoid non-linear distortions even in the case of a highly linear power amplifier a sufficient input-backoff (IBO) of the transmit signal to the amplifier has to be used. Even though the clipping probability of the OFDM signal can be reduced or even be avoided for an IBO larger than the PAR a system with large energy efficiency loss results. Alternatively, the effects which a non-linear device can have on the transmit power spectrum can be influenced by a reduction of the OFDM transmit signal PAR. Different techniques have been developed which reduce the PAR of the OFDM signal by the means of a modified channel coding [5], an additive [6] or multiplicative [7] correction function or a selective mapping of modulation symbols to subcarriers [8]. A majority of these techniques has a high computational complexity due to the fact that they analyze the generated transmit signal and modify it either in the frequency or time domain to reduce its peak amplitudes. For a system employing coherent subcarrier modulation the PAR can also be reduced if the data symbols are spread by a Fourier matrix in frequency direction [3]. 


\section{RESULTS}

We consider an OFDM-FDMA system with $N_{C}=512$ subcarriers and a guard interval of $N_{G}=64$ shared between $M=16$ users. The continuous-time transmit signal $s(t)$ is simulated by a discrete-time signal oversampled by a factor of $K=8$ and bandlimited by a root-raised cosine Nyquist filter. The oversampled and filtered signal is then passed through a non-linear device modelled by a soft-limiter as it has been described in Section IV. The distorted signal $\tilde{s}(t)^{(m)}$ is then analyzed by measuring its power spectral density (PSD) and the its clipping probability. Since only the transmit signal is analyzed in this paper no assumption about the channel have to be made.

\section{A. Out-of-band Radiation}

To evaluate the impact of the proposed OFDM-FDMA transmitter structure on the out-of-band emission after a nonlinear device the power spectral density $\Phi_{s s}^{(m)}(j \omega)$ of the distorted transmit signal $\tilde{s}(t)^{(m)}$ of each user is calculated. Fig. 7 shows the PSD of the used subcarrier for an OFDMFDMA uplink using a DFT-spreading matrix for an IBO of 6 and $9 \mathrm{~dB}$. For comparison the PSD of a conventional OFDM system passed through the same non-linear device is shown. It can be concluded from this plot that the power efficiency can be improved by more than $3 \mathrm{~dB}$ with this approach.

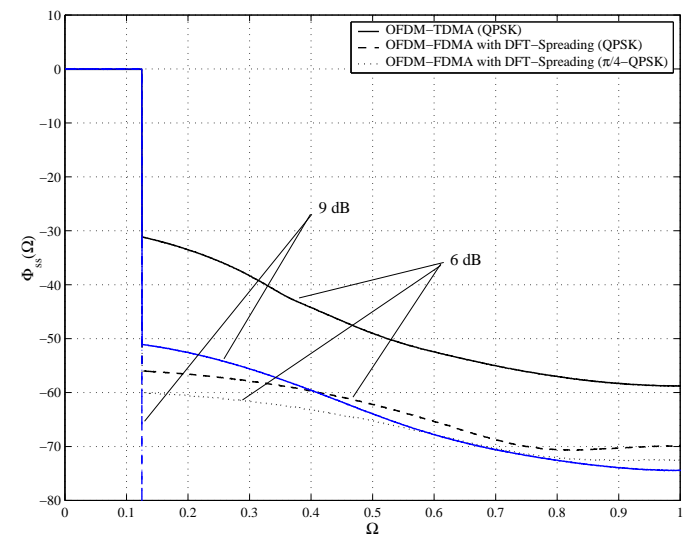

Fig. 7. Power spectral density of a conventional OFDM system and an OFDM-FDMA system for the uplink at the output of a soft limiter

Note that for an OFDM-FDMA system with DFT spreading the PAR and therefore the non-linear distortions are independent from the number of subcarriers per user whereas for a conventional OFDM-FDMA the it is not. The gain in transmit power is therefore influenced by the subdivision of the system bandwidth among the different users. While for a conventional OFDM system the non-linear distortion is independent from the used modulation scheme for the OFDMFDMA uplink with DFT spreading it depends on the PAR of the employed modulation scheme.

\section{B. Amplitude Distribution}

The probability that the instantaneous power $|s(t)|^{2}$ exceeds the threshold $A_{0}^{2}$ is depicted in Fig. 8 versus the normalized threshold. As can be seen from this Figure the power

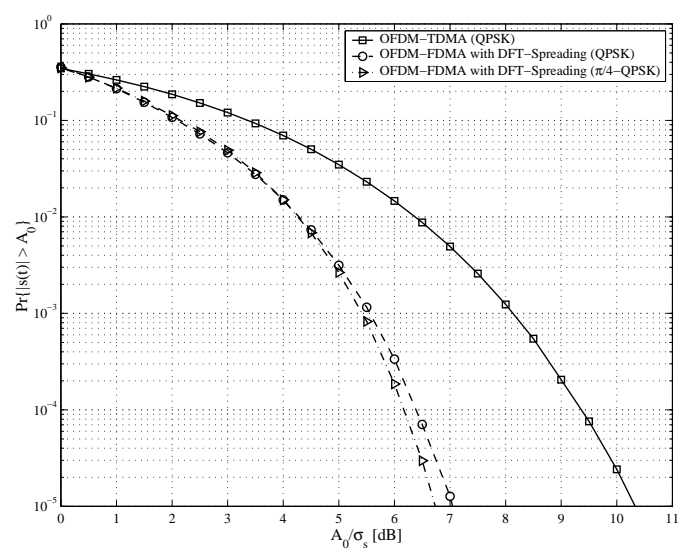

Fig. 8. Clipping probability of a conventional OFDM system and an OFDMFDMA system for the uplink versus the signal input backoff to a soft limiter

efficiency can be increased by $3.6 \mathrm{~dB}$ for the OFDM-FDMA uplink with DFT spreading and a QPSK modulation and by an additional $0.3 \mathrm{~dB}$ for a $\pi / 4$-QPSK for a probabilistic PAR of $10^{-5}$.

\section{SUMMARY}

In this paper an OFDM-FDMA system concept for the uplink of a multi user communication system has been studied. It had be shown that if an OFDM-FDMA system with equidistant subcarrier allocation is combined with a user specific spreading using an discrete Fourier transform as a spreading matrix the transmitter structure can be greatly simplified without any performance degradation. Moreover, the peak-to-average ratio of the transmit signal is reduced to the PAR of the subcarrier modulation scheme which can limit the out-of-band and in-band emissions when the transmit signal is passed through a non-linear device. With the opportunity to independently adjust the user data rates the proposed technique offers an interesting alternative for reducing the complexity of the mobile terminal in the broadband radio uplink.

\section{REFERENCES}

[1] J. A. Bingham, "Multicarrier modulation for data transmission: An idea whose time has come," IEEE Communications Magazine, 1990.

[2] H. Rohling and R. Grünheid, "Performance comparison of different multiple access schemes for the downlink of an OFDM communication system,"' in Proc. of the IEEE VTC, Phoenix, USA, 1997.

[3] K. Brüninghaus and H. Rohling, "Multi-carrier spread spectrum and its relation to single carrier transmission," in Proc. of the IEEE VTC'98,Ottawa, Canada, 1998.

[4] J. Park, J. Kim, S. Choi, N. Cho, and D. Hong, "Performance of MCCDMA systems in non-independent rayleigh fading," in Proc. of IEEE ICC'99, Vancouver, Canada, 1999.

[5] A. Jones and T. Wilkinson, "Cobined coding for error control and increased robustness to system nonlinearities on OFDM,',in Proc. of IEEE VTC'96, Atlanta, USA, 1996.

[6] T. May and H. Rohling, "Reducing the peak-to-average power ratio in OFDM radio transmission systems," in Proc. of the IEEE VTC, Ottawa, Canada, 1998.

[7] M. Pauli and H.-P. Kuchenbecker, "Reduzierung der durch Nichtlinearitäten hervorgerufenen Aus̈erbandstrahlung bei einem Mehrträgerverfahren," ITG Fachberichte 135 "Mobile Kommunikation”, 1995. (In german).

[8] M. Breiling, S. H. Müller-Weinfurtner, and J. B. Huber, "Peak-power reduction in OFDM without explicit side information," in Proc. of the 5th Int. OFDM-Workshop (InOWo'00), Hamburg, Germany, 2000. 